\title{
The ESPA (Enhanced Structural Path Analysis) method: a solution to an implementation challenge for dynamic life cycle assessment studies
}

\author{
Didier Beloin-Saint-Pierre • Reinout Heijungs • \\ Isabelle Blanc
}

Received: 25 July 2013 / Accepted: 21 January 2014 / Published online: 4 February 2014

(C) The Author(s) 2014. This article is published with open access at Springerlink.com

\begin{abstract}
Purpose By analyzing the latest developments in the dynamic life cycle assessment (DLCA) methodology, we identify an implementation challenge with the management of new temporal information to describe each system we might want to model. To address this problem, we propose a new method to differentiate elementary and process flows on a temporal level, and explain how it can generate temporally differentiated life cycle inventories (LCI), which are necessary inputs for dynamic impact assessment methods.

Methods First, an analysis of recent DLCA studies is used to identify the relevant temporal characteristics for an LCI. Then, we explain the implementation challenge of handling additional temporal information to describe processes in life cycle assessment (LCA) databases. Finally, a new format of temporal description is proposed to minimize the current implementation problem for DLCA studies.

Results and discussion A new format of process-relative temporal distributions is proposed to obtain a temporal differentiation of LCA database information (elementary flows and product flows). A new LCI calculation method is also proposed since the new format for temporal description is not compatible with the traditional LCI calculation method. Description of the requirements and limits for this new
\end{abstract}

Responsible editor: Hans-Jörg Althaus

Electronic supplementary material The online version of this article (doi:10.1007/s11367-014-0710-9) contains supplementary material, which is available to authorized users.

D. Beloin-Saint-Pierre $(\bowtie) \cdot$ I. Blanc

OIE, MINES ParisTech, 1 rue Claude Daunesse, 06904 Sophia

Antipolis, France

e-mail: dbstp81@gmail.com

R. Heijungs

Department of Econometrics \& Operations Research, VU University

Amsterdam, Amsterdam, The Netherlands method, named enhanced structural path analysis (ESPA), is also presented. To conclude the description of the ESPA method, we illustrate its use in a strategically chosen scenario. The use of the proposed ESPA method for this scenario reveals the need for the LCA community to reach an agreement on common temporal differentiation strategies for future DLCA studies.

Conclusions We propose the ESPA method to obtain temporally differentiated LCIs, which should then require less implementation effort for the system-modeling step (LCA database definition), even if such concepts cannot be applied to every process.

Keywords Dynamic life cycle assessment (DLCA) . Enhanced structural path analysis (ESPA) $\cdot$ Life cycle inventory (LCI) · Temporal database description · Temporal differentiation methodology

\section{Introduction}

Since their inception, most life cycle assessment (LCA) studies have considered few system variations over time and a static environmental response to extractions and emissions. In addition, they usually aggregate all elementary flows over the entire life cycle (Finnveden et al. 2009), thereby preventing any explicit temporal differentiation.

In the last 15 years, however, compelling arguments have been proposed to explain why industrial and environmental dynamics might have significant impacts on the results of some LCA studies (Field et al. 2000; Finnveden et al. 2009; Graedel 1998; Owens 1997; Reap et al. 2008; Udo de Haes et al. 2002). Indeed, not considering temporal variability is now recognized as one of the shortcomings of the LCA methodology (ISO 14040 and 14 044). This gap between 
expectations of dynamic consideration and current static implementation of the LCA methodology needs to be bridged to increase the representativeness for results of future LCA studies.

In the last few years, many dynamic LCA (DLCA) studies have pursued this goal and shown the relevance of considering time for some systems and environmental impacts. Among those publications, we distinguish two categories of discussions, which relate either to impact assessment or system modeling.

\subsection{Time considerations for environmental impact assessment}

Reap et al. (2008) have listed many examples of how impacts might vary if the rate or timing of emissions changes. In their review, they underline that Owens (1997) acknowledged that the state of the environment and the rate of release (flow) of pollutants might affect the level of impacts from emissions at certain times.

Many other examples have been proposed to demonstrate the importance of emission timing. Graedel (1998) stressed that a certain amount of volatile organic compounds released during daylight will produce more photo-oxidants than the same amount released over an entire day. Udo de Haes et al. (2002) explained how the acidification impacts change when an ecosystem's nitrogen holding capacity is exceeded. Looking more specifically at impact factors, Shah and Ries (2009) have shown how the fate level characterization of $\mathrm{NO}_{\mathrm{x}}$ can vary by about two orders of magnitude between emission in summer or winter across different states of the USA.

The importance of time horizons has also been taken on board in the development of a dynamic impact assessment method for climate change created by Levasseur et al. (2010). Using this new impact assessment method, one case study has shown that conclusions from an LCA study might significantly vary when different time horizons are considered. Other publications (Dubreuil et al. 2007; Field et al. 2000; Hellweg 2001; Kendall and Price 2012; Kendall 2012) have presented similar conclusions even though some authors (Schwietzke et al. 2011) minimize its relevance for biofuel scenarios.

The Shonan principles (Sonnemann et al. 2011) have acknowledged the previous observations and proposed a list of impact categories which might vary significantly as a function of time. In those principles, water withdrawal and consumption, land-use GHG emissions, or photochemical oxidants creation potential (POCP) are the impacts categories considered as time sensitive.

1.2 Considering time for system modeling and life cycle inventory calculation

Specific mathematical models have been provided to account for a few temporal systems variations in different DLCA studies (Bjork and Rasmuson 2002; Collinge et al. 2011; Field et al. 2000; Kendall et al. 2009; Pehnt 2006; Stasinopoulos et al. 2012; Zhai and Williams 2010). Such models usually describe how parts of systems vary over the life cycles.

The life cycle inventories (LCIs) obtained in those studies are different from those of more traditional LCA studies where process flows do not evolve during the life cycle of their systems. Those examples, considering the process variation over time, cause some modifications to the conclusions, which indicate that industrial dynamics can have an effect on the results of DLCA studies. From what we could gather, most of those time-dependent LCIs are not explicitly differentiated at the temporal level, which precludes the use of time-dependent impact factors with the obtained "dynamic" LCI.

\subsection{Complete dynamic life cycle assessment studies}

Recently, Collinge et al. (2013) have used a temporal differentiation method developed by Heijungs and Suh (2002) to model their system. They then used a calculation structure proposed by Mutel and Hellweg (2009) in order to explicitly describe the temporal variability in the LCI obtained. It is the only study we could find where time is explicitly taken into account in both LCI calculation and impacts assessment phases.

1.4 The next implementation challenge to take time into account in DLCA studies

When we looked more closely at the description of the method used by Collinge et al. (2013) and developed by Heijungs and Suh (2002), we find that the authors clearly underline an implementation challenge by stating that: "the extent to which differentiation is feasible will be quite restricted in practice." This is explained by the expected increase in data required to describe the temporal variation of different systems in LCA databases. And so, while complete DLCA studies are now clearly possible, there still seems to be an implementation challenge due to the management of temporal information for system description and modeling. This is why we propose a specific three-step strategy to address this issue.

\section{Development strategy to solve the implementation challenge}

The first step to propose a solution to the raised implementation issue is to clearly define the temporal characteristics that are useful for environmental impact assessments (required inputs). In the second step, we analyze in detail the method available today (Heijungs and Suh 2002) and describe, with 
an example, why temporal differentiation is restricted in practice. In the third and final step, we identify the current characteristics of process definition and explain why it is not subjected to the same implementation restriction. Based on this three-step strategy, we then propose a new temporally explicit method that we call enhanced structural path analysis (ESPA).

\subsection{Temporal inputs required for impact assessment}

To suggest an explicit description of the temporal variation for a scenario, we first look at the results format that would be useful for an impact assessment. In the work of Levasseur et al. (2010), the dynamic impact assessment requires that life cycle emissions be described through distributions. This underscores the need to temporally differentiate all elementary flows of a supply chain over its life cycle through discrete temporal distributions.

The dynamic impact assessment method developed by Levasseur et al. (2010) also calls for a temporal description which is relative to a chosen time horizon. When we analyzed other temporally sensitive impact categories, it became clear that a seasonal calendar-related description might also be useful. For example, water depletion effects are not related to the start of a life cycle but more to the season. This is why we think that a temporal differentiation of LCI would need to temporally describe elementary flows in relation to our calendar. The time horizon could then be set relative to a specific date.
Looking at different impact categories also brings the issue of accuracy in temporal differentiation. Most current DLCA studies have arbitrarily chosen an annual time step for temporal description, but seasonal or daily variations could be useful in some cases. A temporal differentiation method that can model any system should make use of different temporal precision and would probably be needed for future dynamic impact assessment methods. As another example, impact categories such as noise (Cucurachi et al. 2012) are not specifically explained in our new method, but they would require a differentiation of elementary flows between day and night.

To summarize, our analysis of the required temporal input for dynamic impact assessments has brought forth three criteria for LCI temporal differentiation. This differentiation will need to be calendar relative, defined with temporal distributions and different accuracies. Reaching those criteria for a temporally defined LCI is the first requirement we identified for our approach.

\subsection{Current temporal differentiation method for a system description}

More than 10 years ago, Heijungs and Suh (2002) described a method to temporally differentiate the process of any database. The idea is comparable to the one used to differentiate flows on a spatial basis. The following simplified technological product-by-process matrixes describe how electricity and coal production process flows can be disaggregated for two different years (2001 and 2002 in this case).

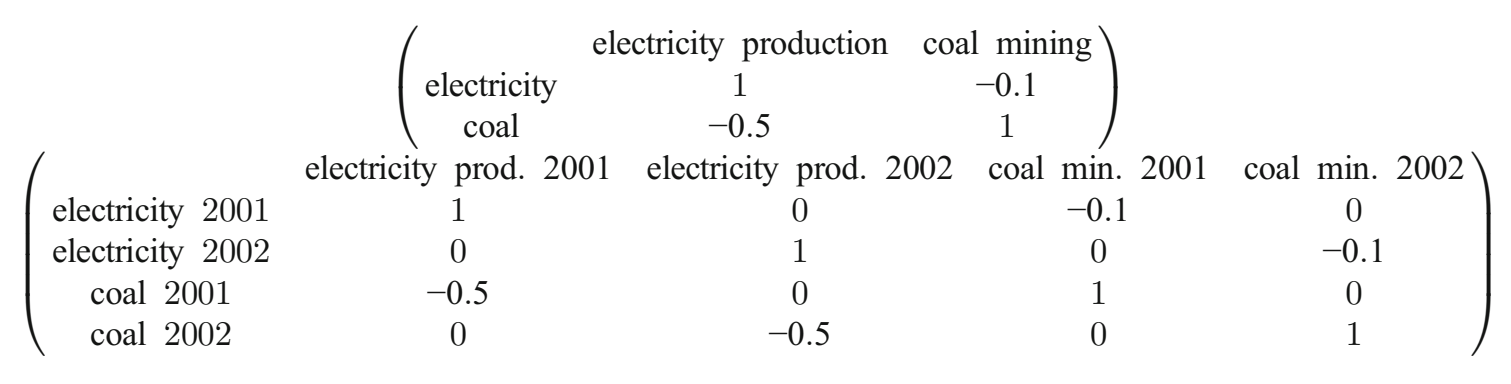

This method can create temporal distributions of elementary flows (if applied to the environmental matrix) with annual accuracy when each flow represents a discrete year. In fact, it gives a calendar-relative temporal distribution, where temporal differentiation can vary in accuracy. By doing so, it meets all the criteria we identified in the last subsection (2.1). Its limited practical use, mentioned in Section 1, is still an issue, and this implementation challenge is still to be solved. To find a solution, we now describe, in detail, how Heijungs and Suh's method is not practical for the information structure used in the current LCA studies.
Today's LCA databases can describe thousands of processes. Using Heijungs and Suh's method to temporally differentiate all of those processes with different accuracies would mean an unmanageable increase in the amount of process and elementary flows to be defined. To clarify, let us take a simplified example where we compare the environmental impacts of the production of a solar energy installation with the environmental impacts of the electricity produced in a country. Many solar energy enthusiasts will say that for a fair comparison, we should only consider the period of time when a solar system is producing electricity (a certain number of hours per 
day). This would mean an hourly process differentiation over the lifetime of the system, which is about 30 years. So, for this specific comparison, our electricity production would require the definition of up to $262,800(30 \times 365 \times 24)$ processes. This is, at least, two orders of magnitude above current database sizes, and it does not consider the temporal differentiation of elementary flows. To add to the complexity, a calendar-specific differentiation would only work for one study since process flows are case specific with this method. The same study made 10 years later would require the temporal definition of the same number of processes since the temporal description is case specific. For both those reasons, we can see the practical limit of such a temporal differentiation method.

\subsection{Current characteristics of a process definition in a LCA database}

The previous description of the only available temporal differentiation method highlights how implementation will be an issue for LCA practitioners and database managers. So, the main challenge is to find a way of minimizing the increase in data imposed by this temporal differentiation. In many traditional LCA studies, the large amount of data that needs to be considered has been handled by the reuse of the same case for processes throughout a system description. This reusability of processes is possible because of the relative nature of defined elementary and process flows. In other words, the flows defined in a dataset are relative to the process and, with scaling, those flows can describe other equivalent processes occurring at a different place in the system. This relative nature of the information we use today is key to minimizing the amount of data to be managed. Our working hypothesis is that a solution to the implementation challenge of temporal differentiation will require such a process-relative description of time.

\section{New temporal differentiation method}

Based on our overview of the main challenges and requirements of current dynamic LCA (DLCA) studies, we agree with the proposition made by Collinge et al. (2013) that this type of study requires addressing explicitly the temporal characteristics, both for the system modeling and for the environmental impact assessment phases. Our research has focused on the explicit inclusion of temporal information to model the systems and how it can affect the calculation of the LCI.

Our suggestion for an explicit temporal differentiation in system modeling (database information) is to use processrelative temporal distributions to describe both elementary and process flows. Here, the term "distribution" is linked to the theory developed by Laurent Schwartz and is also called the generalized function (Schwartz 1950). From those distributions, we can obtain temporally differentiated LCIs which respect all of the identified criteria (see Subsection 2.1) for the use of a dynamic impact assessment method. In the following paragraphs, we go over the details of defining process-relative temporal distributions and how to calculate a temporally explicit LCI.

\subsection{Definition of process-relative temporal distributions}

A process-relative temporal distribution describes flows per unit of time (in other words, a rate of process). Table 1 presents four different examples of process-relative temporal distributions to describe temporally differentiated process flows linked to the description of processes A to D. In a similar manner, Table 2 presents three temporal distributions, which describe the elementary flows (rate of emissions) relative to the description of processes $\mathrm{A}$ to $\mathrm{D}$.

In those examples of process-relative distributions, the $x$ axis is divided according to the temporal accuracy (monthly or yearly). Here, a different precision could be used to describe different flows or even one flow. The $y$-axis gives the amount of flow per unit of time (rate which depends on the temporal accuracy on the $x$-axis).

The distributions we use must have a compact support. This means that the integral over time for the distribution must be equal to a real number. This real number is equivalent to the measure that would typically describe the elementary or process flow in a traditional LCA database.

The definition of a time zero for process-relative temporal distributions, which describe process or elementary flows, should be standardized with respect to the process in which they are described. We propose this time zero to be the time when the product, service, or system linked to the "parent" process is ready to be used. As an example, time zero for process-relative distributions linked to a power plant description is when the power plant is ready to produce electricity. A standardized setting of time zero is critical for linking processrelative information to the temporal description of a case study. This time zero for elementary flows must also be defined according to the same logic, which means that if we know that building a dam will create emissions of methane 5 years after the dam started producing electricity, the emissions distribution for methane should start 5 years after the time zero of that particular emission's temporal distribution.

\subsection{Temporally differentiated LCI calculation methods}

The process-relative temporal distributions described in the last subsection (3.1) can increase the reusability of data that describe processes in DLCA studies. However, they, alone, cannot offer a case-study specific or a calendar-relative differentiation of LCI. The LCI calculation method must be modified in order to propagate the relative temporal information for a specific life cycle. Two modifications must be made for such propagation. 
Table 1 Process-relative temporal distributions of process-flows linking processes A to D

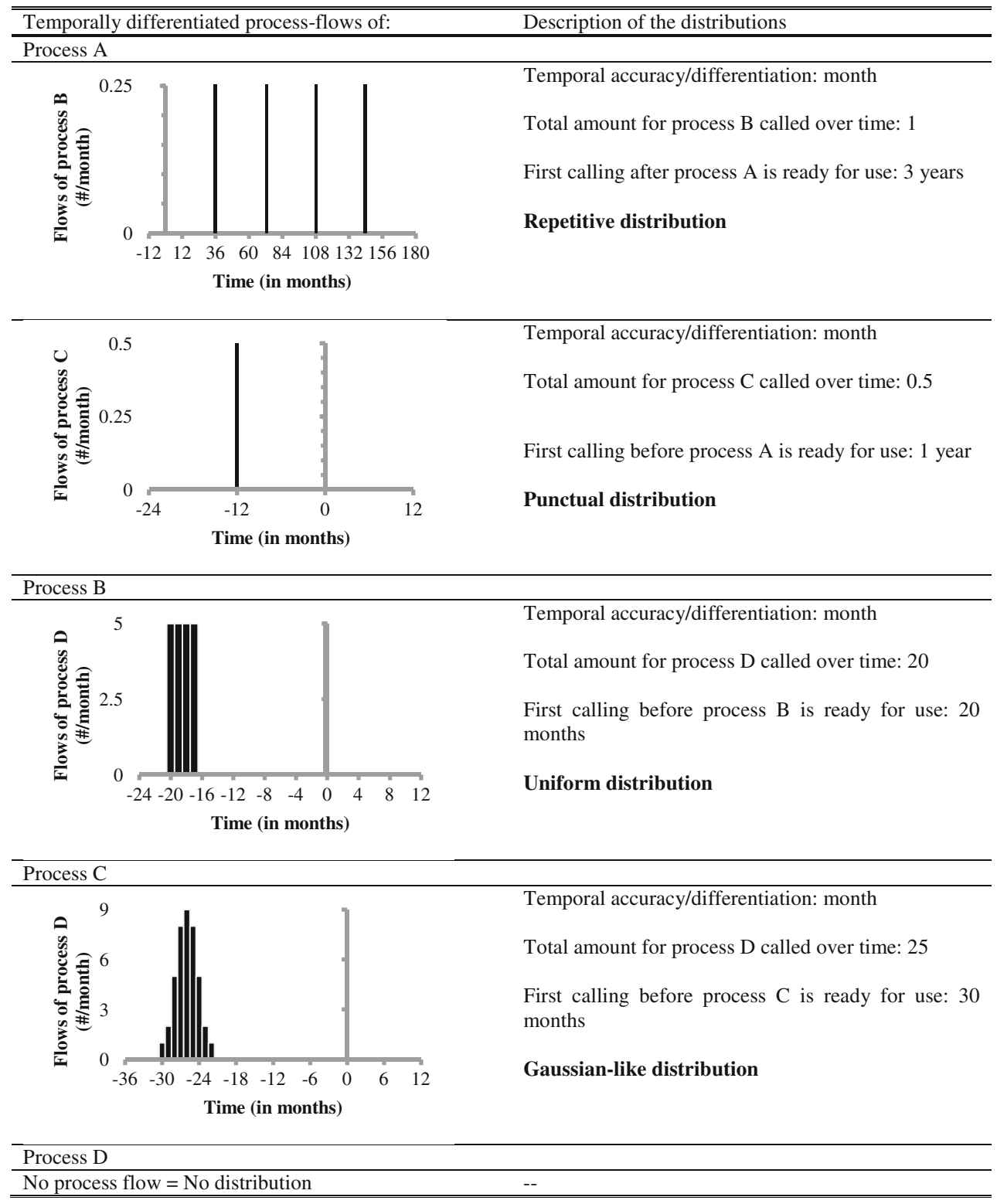

First, we need to propagate the temporal specificities of a case study across the temporal distributions used in the process descriptions. In other words, the starting times for the system processes need to be relative to the full life cycle of the scenario. In the standard LCI matrix calculation method, products would be used between two processrelative temporal distributions, but this mathematical operation will not propagate the temporal information included in the process-relative distributions. We need to use a product of convolution to obtain the temporal information propagation we are looking for. The description of a product of convolution is given in Electronic supplementary material (ESM) 1, but this paper explains how temporal information is propagated.
First, to understand how the product of convolution is used, it is important to note that a linear relationship must exist to use the mathematical operator to propagate temporal information between elements of matrixes. To explain why this relationship must be linear, let us recall the input/output format of the traditional LCI calculation equation:

$\overrightarrow{\boldsymbol{o}}=\mathbf{E} \cdot(\mathbf{I}-\mathbf{T})^{-1} \cdot \vec{r}$

Where:

$\overrightarrow{\boldsymbol{o}}$ is the inventory vector defining the LCI of the scenario linked to the processes defined in vector $\vec{r}$

E is the environmental matrix (or intervention matrix) which defines the elementary flows for any process 
Table 2 Process-relative temporal distributions of elementary flows linked to processes A to D

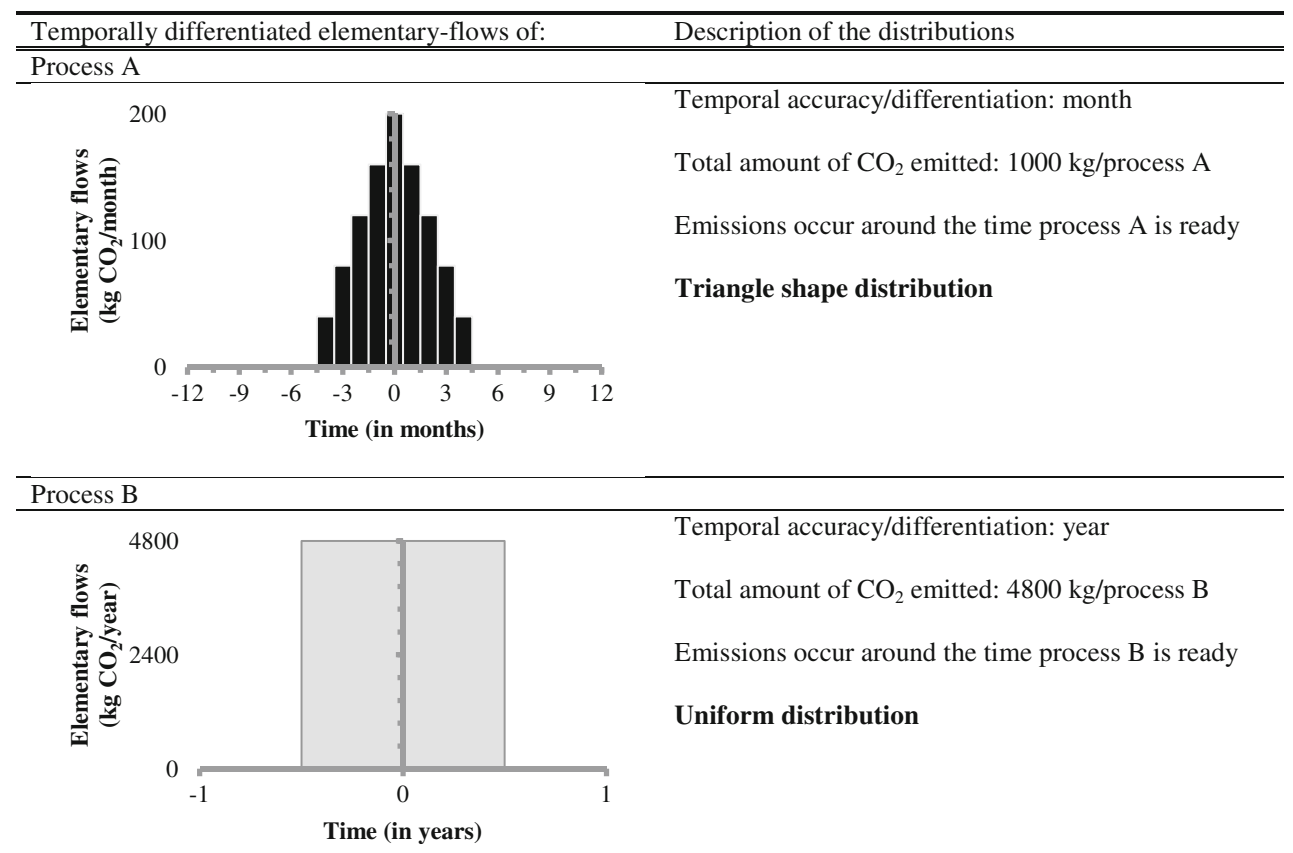

\begin{tabular}{ll}
\hline Process C \\
\hline No emissions = No distribution \\
\hline Process D \\
\hline
\end{tabular}

defined in the matrix $\mathbf{T}$ (traditionally described by the letter B)

$\mathbf{T}$ is the technological matrix which describes the process flows (traditionally described by the letter $\mathbf{A}$ )

$\vec{r}$ is the scenario's vector defining the processes that are directly required to model the scenario

In Eq. 1, the inverse operator applied to the (I-T) matrix does not admit the use of a product of convolution between its elements and the elements of matrix $\mathbf{E}$ and vector $\vec{r}$. We, therefore, had to come up with a second modification to the traditional LCA methodology, inspired by the structural path analysis (Defourny and Thorbecke 1984; Lenzen 2007) and the power series (PS) methods (Suh and Heijungs 2007). Both methods solve Eq. 1 using Taylor's expansion. Equation 1 then becomes:

$$
\overrightarrow{\boldsymbol{o}}=\mathbf{E} \cdot\left(\mathbf{I}+\mathbf{T}+\mathbf{T}^{2}+\mathbf{T}^{3}+\cdots+\mathbf{T}^{j}+\cdots+\mathbf{T}^{n}\right) \cdot \vec{r}
$$

The use of equation 2 requires that the series be stopped at a certain level of the system. In practice, this is not a critical problem since we can use a high enough $n$ level to obtain a result that converges with the result of Eq. 1, as long as the elements of matrix $\mathbf{T}$ respect certain conditions:

1. Linear system modeling

2. The eigenvalues of $\mathbf{T}$ need to have a modulus which is less than unity

3. The norm of (I-T) needs to be less than one $-N(\mathbf{I}-\mathbf{T})<1$.

The previous conditions are fully explained by Suh and Heijungs (2007).

More details are now given to understand how the product of convolution is used in such an equation. Equation 3 presents the traditional calculation for an element $\alpha$ of the $\overrightarrow{\boldsymbol{o}}$ inventory vector representing the emissions of substance $\alpha$ for the third level of a system. 
$o_{\alpha}=\sum_{z} \sum_{y} \sum_{x}\left(e_{\alpha z} \times\left(t_{z y} \times\left(t_{y x} \times r_{x}\right)\right)\right)$

Where:

$o_{\alpha} \quad$ is the amount of $\alpha$ substance linked to the first three levels of the system defined by $\vec{r}$

$z \quad$ is a summation index over the elementary flows for all the processes defined in $\mathbf{T}$

$y$ and are summation indexes over all the processes defined

$x \quad$ in $\mathbf{T}$ and $\overrightarrow{\boldsymbol{r}}$

$e_{\alpha z} \quad$ is the elementary flow of matrix $\mathbf{E}$ for the $\alpha$ row and $z$ column

$t_{z y}$ is the process flow of matrix $\mathbf{T}$ for the $z$ row and $y$ column (the same logic applies to $t_{y x}$ )

We then modify Eq. 3 to use process-relative temporal distributions instead of values for each of the elements and replace the products by products of convolution:

$o_{\alpha}=\sum_{z} \sum_{y} \sum_{x}\left(e_{\alpha z} *_{\text {temp }}\left(t_{z y} *_{\text {temp }}\left(t_{y x} *_{\text {temp }} r_{x}\right)\right)\right)$

$*_{\text {temp }}$ is the symbol we use to define a product of convolution on the temporal dimension of the distributions. This means that the product of convolution is only applied to the distributions and not between matrices. As said previously, the product of convolution is the operator that propagates the process-relative temporal information onto a specific system's life cycle. The propagation of information is quickly explained in ESM 1, but a more complete example is presented in Subsection 3.3.

Equation 4 details explicitly how we modified the LCI calculation method in order to use a process-relative temporal distribution to obtain an element of the temporally differentiated LCI which meets the criteria we had previously identified in Section 2.1. A general definition of the equation to calculate the inventory is, therefore, expressed in Eq. 5:

$\overrightarrow{\boldsymbol{o}}=\mathbf{E} *_{\text {temp }}\left(\mathbf{I}+\mathbf{T}+\mathbf{T} *_{\text {temp }} \mathbf{T}+\mathbf{T} *_{\text {temp }} \mathbf{T} *_{\text {temp }} \mathbf{T}+\cdots\right) *_{\text {temp }} \overrightarrow{\boldsymbol{r}}(5)$

where we propose that the use of process-relative temporal distributions and the modification of the LCI calculation be called the enhanced structural path analysis (ESPA) method (Beloin-Saint-Pierre and Isabelle 2011a, b).

3.3 A strategic case study for the implementation of the ESPA method

The scenario used as example consists of four interlinked processes and their respective $\mathrm{CO}_{2}$ emissions (elementary flows) defined by process-relative temporal distributions. The objective is to assess the temporal distribution of $\mathrm{CO}_{2}$ emissions related to process $\mathrm{A}$ (functional unit $=$ one process
A, which also defines the vector $\vec{r}$ ). The characteristics of this made-up scenario have been strategically chosen to show important aspects to be considered in the temporal differentiation of database and LCI.

Figure 1 presents the supply chain of our simplified case study up to the third level. In Fig. 1, the process flows are identified as thin black lines. The hollow white arrows represent $\mathrm{CO}_{2}$ emissions from each process within the system. There is no white line coming from process $\mathrm{C}$ since this process does not emit any $\mathrm{CO}_{2}$. Numbers 1 to 4 will serve to simplify the identification of an emission structure in the full life cycle temporal distribution of the scenario.

Table 1 (see Subsection 3.1) describes process flows (elements of matrix T). Table 2 (see also Subsection 3.1) gives the $\mathrm{CO}_{2}$ emissions (elementary flows) for all those processes (elements of matrix $\mathbf{E}$ ). All process-relative temporal distributions (shown in Tables 1 and 2) meet the previously identified requirements for temporal distributions. This means that the integrals over time of those distributions are equivalent to real values, and the time zero position is relative to their respective processes.

We use Eq. 6 to calculate $\mathrm{CO}_{2}$ emissions (the only element of the inventory vector $\overrightarrow{\boldsymbol{o}}$ in this example) for the supply chain described by the distributions of Tables 1 and 2. The Taylor development is applied up to the third level as our scenario consists only of three levels. The $\mathbf{T} \overrightarrow{\boldsymbol{r}}$ vector in the last element of Eq. 6 is a simplified representation of the $\mathbf{T}$ ${ }_{\text {temp }} \vec{r}$ calculation of the second element in the same equation. We use this presentation format to clearly show that our calculation method meets the need for a linear relationship when calculating the product of convolution on the temporal dimension of different matrixes.

$\overrightarrow{\boldsymbol{o}}=\mathbf{E} *_{\text {temp }} \overrightarrow{\boldsymbol{r}}+\mathbf{E} *_{\text {temp }} \mathbf{T} *_{\text {temp }} \overrightarrow{\boldsymbol{r}}+\mathbf{E} *_{\mathrm{temp}} \mathbf{T} *_{\mathrm{temp}} \mathbf{T} \overrightarrow{\boldsymbol{r}}$

The result of Eq. (6) is given in Fig. 2 as a temporal distribution over the case study's lifetime. Setting time zero

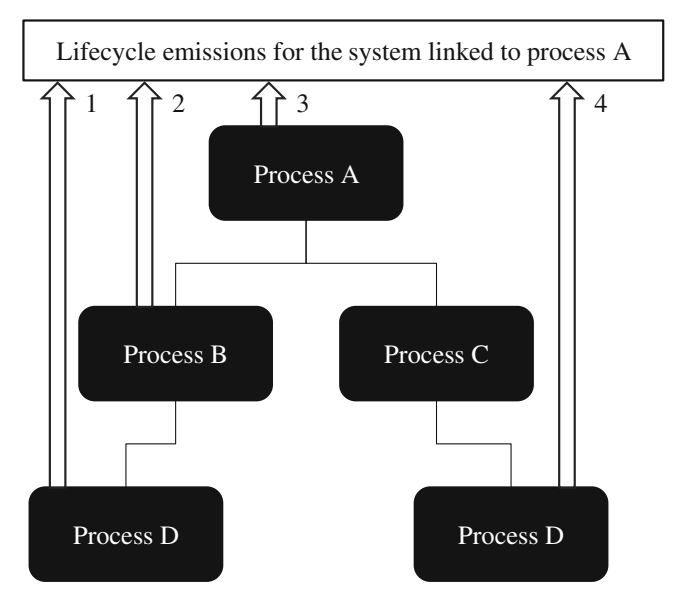

Fig. 1 Tree representation of the system for the example studied 
Fig. 2 Temporal distribution of $\mathrm{CO}_{2}$ emission over the entire life cycle for the example studied

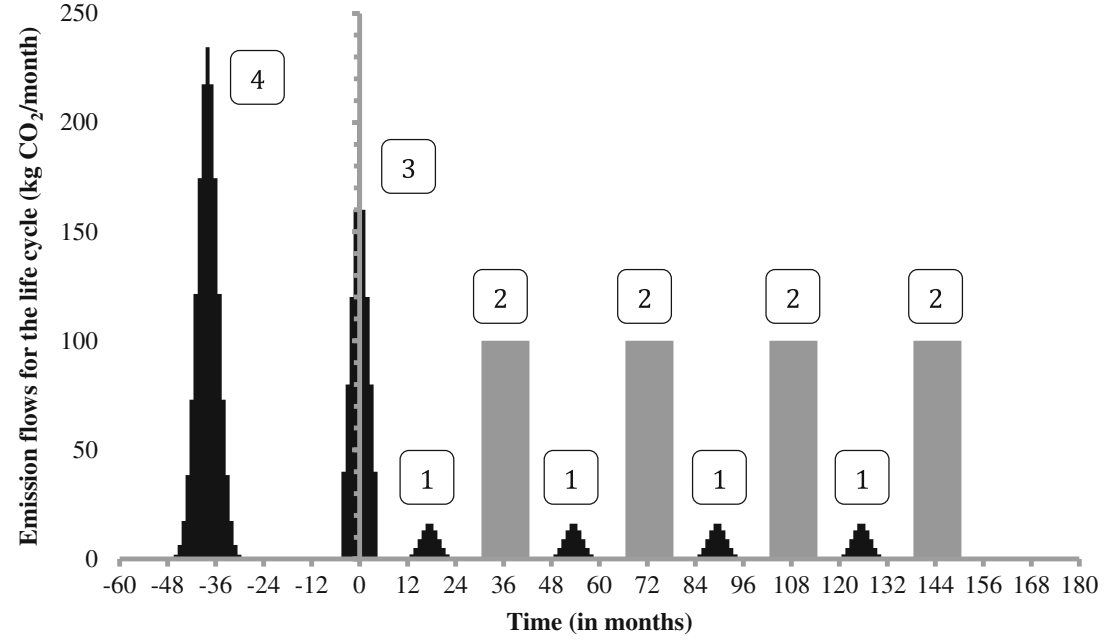

of process A to January 2013 would then create a calendarrelative temporally differentiated LCI result. It represents, in visual form, the distribution of the LCI data that will be available for the impact assessment step of the LCA study for one process A. Numbers in Fig. 2 are linked to the description of the system's elementary flows in Fig. 1.

An easily identifiable clue indicating that the scenario's emissions are correctly modeled is the recurring structure of called process B (described in Table 1) which can be observed in Fig. 2 with both small Gaussian-like (process B calling emissions from process D) and rectangular functions (emissions directly related to the calling of process B). The result of Fig. 2 shows that we can reuse the same process relative definition at different times in a scenario's lifecycle.

Time zero for the full life cycle of the system is the moment when process A can be used. And so, we can easily identify this scenario's past and future emissions when using one process A. When looking at the numbered emissions in Fig. 1, we can link the first peak of final temporal distribution to emissions from process $\mathrm{D}$ (arrow \#4) called by process $\mathrm{C}$. The second peak describes emissions coming directly from process A (arrow \#3). The four small peaks describe the emissions (arrow \#1) from process D called by process B. The four large rectangles describe the emissions flows coming directly from process B (arrow \#2).

In this particular case, certain structures are in gray since they show a yearly rather than a monthly precision. This difference needs to be well documented to use the data more accurately once more dynamic impact assessment methods become available, in order to take into account the effect of varying emission flows in the environment.

When the flows' temporal accuracy varies between calculation steps, it is important to keep the information about the loss of accuracy for subsequent levels of the supply chain. In our example, the accuracy loss is directly related to emissions and does not affect the precision of subsequent emissions, but the problem may arise if the loss of temporal accuracy is related to process flows. At this time, we do not know the acceptable level of temporal accuracy we should use to ensure a representative level for any impact assessment method. This means that a maximum level of temporal precision will be more useful to guaranty the future applicability of a scenario description in a database. Hence, we believe that it is important to try and reach the highest precision for a process flow description.

The result presented in Fig. 2 can be verified by integrating the final distribution over the entire life cycle. The integration results must give a value equivalent to the value that would be obtained if we made a traditional LCI calculation with only total amounts in the description of distributions columns of Tables 1 and 2. In this case, for both calculation methods, the full consolidated life cycle $\mathrm{CO}_{2}$ emission for the scenario is equivalent to $7,712.5 \mathrm{~kg}$ over the lifetime considered ( 200 months).

\section{Discussion}

We have discussed temporal differentiation in the steps of system modeling and LCI calculation (phase 2 of ISO 14 040 structure). Using the ESPA method in the context of our example brings different observations and requirements for both steps.

\section{Step 1 Scenario description}

When looking at the size and quantity of information already needed for traditional databases, it becomes clear that temporal differentiation of LCA scenarios would impose an important workload. One of our goals was to find a way of minimizing this effort through "wise" temporal descriptions. We believe that using process-relative temporal distributions to model elementary and process flows will 
scale down the required effort since our method of describing process-based scenarios enables us to reuse certain process definitions throughout a system.

On the other hand, if we want to use our description for temporal information of scenarios, it is important to understand that temporally differentiating every process is not a prerequisite. However, missing a temporal link within the system will prevent the temporal differentiation of all related subprocesses. Using Boolean flags to identify information with no temporal differentiation would allow part of the calculation to be made with the traditional LCI calculation. Any subprocess described by process-relative temporal distribution would then be integrated over time and defined by a single numerical value. This means that before LCA databases are fully temporally differentiated, other techniques like that proposed by Collet et al. (2011) can be quite useful to identify part of the supply chain which should be prioritized on a temporal level and start partial analyses.

The ESPA method will not systematically minimize the efforts needed since the required invariability by time translation of the process does not apply to every case. The description of an infrastructure is a clear example where the ESPA method does not decrease the work needed for a temporal differentiation. The difficulty when modeling infrastructure is that life cycle impacts from this part of the system are set at a specific time, regardless of the timing for the study. The example of the LCA for electricity production scenarios highlights this difficulty since most impacts from electricity production are temporally linked to the times when electricity is produced, except for the impacts of the power plants themselves. In this subsection of the system, impacts from power plants will always be set in relation to the time of construction, regardless of the time when electricity is produced. In such cases, a calendar-specific definition of the system will be required to make the temporal link with time zero for the infrastructure.

Looking at how we have described the temporal characteristics of systems, it becomes clear that certain rules will be required if the community wants to exchange a temporally defined datasets. The setting of time zero is a good example of how a different definition would cause important problems in the reusability of different sources of information. More case studies will be required to see if certain activity datasets cannot use the "ready-to-be-used" rule, but we have not found any so far. We, therefore, want to stress the need for discussions between experts on this subject to investigate any possible shortcomings of this approach while conducting larger case studies.
Such work should be done rapidly since it is a prerequisite to start temporal differentiation of databases, and the more informed data we gather today, the more temporally representative the future databases will be.

Step 2 LCI calculation and format

The calculation of a temporally differentiated LCI with the ESPA method means that we will stop the system modeling at a certain level. This could cause problems mostly in terms of calculation time for scenarios where most of the impacts are coming from background data. It would, in that case, require longer series to consider an important proportion of the impact over the life cycle. In practice, many tests will be required on different systems to see if we can find large differences in life cycle impact evaluation, caused by a truncated system. Interesting work in relation to systematic disaggregation has been presented by Bourgault et al. (2012). The findings of this particular work could also be useful in minimizing the size of the technosphere matrix at each level of recursion and helping in the management of temporal description. Further test will, however, be needed to evaluate how this could be done.

The LCI format based on temporal distributions we propose is in direct correspondence with our analysis of required inputs for dynamic impact assessment methods, such as the one created by Levasseur et al. (2010). This means that we can calculate the life cycle elementary flows at different times and with different accuracies for different systems. We could also present information in another format, if it were more useful to evaluate certain impacts. For example, we could give the accumulation of a substance over the life cycle if we know its site and related environmental diffusion mechanisms. This could be useful when looking at threshold effects of certain substances. The different possibilities for formats of LCI results highlight the need for a discussion with designers of impact analysis methods in order to identify where LCI calculation should stop and where impact analysis should begin in the LCA methodology.

\section{Conclusions}

In this paper, we propose the ESPA method to temporally describe elementary and process flows and calculate relevant temporally differentiated LCI. The main purpose of this method is to decrease the implementation workload linked with DLCA studies.

The ESPA method decreases the workload for the description of time in scenarios because we can reuse many temporally defined processes in different systems and even within a single 
system. This is possible since the ESPA LCI calculation method propagates process-relative temporal characteristics throughout the different levels of the scenario's system. However, developing databases is a joint effort of the LCA community, and a discussion on the use of process-relative temporal distributions is needed to reach an agreement on some aspects of the format. A faster switch to a common format will increase our future ability to take time into account and carry out more DLCAs.

The temporally differentiated LCI we can obtain with our method could be used with previously proposed dynamic impact assessment methods, (Levasseur et al. 2010) but results could be presented in a different format to help with other impact categories.

The evaluation of the importance of time characterization on final LCA results will require further studies with timedependent impact assessments. To reach this goal will still probably mean a considerable workload, for one main reason: today, LCA databases (ecoinvent, ELCD, GaBi) offer little temporal information. In fact, the only temporal information is the time representativeness of a defined process. This means that determining the various time lags between processes and elementary flows will require an additional amount of work. The temporal differentiation of process flows will probably require more work than temporal differentiation of elementary flows because more accurate information for the former will ensure a broader use in the modeling of different systems.

Our next step is to work in collaboration with other researchers on the application of the ESPA method to make DLCA studies of complex systems and look at the effect of time on results and analysis.

Acknowledgement The authors wish to acknowledge the financial support of MINES ParisTech for the research on dynamic LCA. We would also like to thank the two anonymous reviewers and Christine Groslambert-Malins for their valuable inputs on the final manuscript. Finally, we thank Annie Levasseur, Manuele Margni, and Pascal Lesage from CIRAIG and Philippe Blanc from MINES ParisTech for their useful feedback on the preliminary work.

Open Access This article is distributed under the terms of the Creative Commons Attribution License which permits any use, distribution, and reproduction in any medium, provided the original author(s) and the source are credited.

\section{References}

Beloin-Saint-Pierre D, Isabelle B (2011a) New spatiotemporally resolved LCI applied to photovoltaic electricity, LCM-Towards Life Cycle Sustain Manag. Berlin

Beloin-Saint-Pierre D, Isabelle B (2011b) Enhanced structural path analysis: a new method to create spatiotemporally defined life cycle inventory. SETAC Eur Annu Meet, Milan
Bjork H, Rasmuson A (2002) A method for life cycle assessment environmental optimisation of a dynamic process exemplified by an analysis of an energy system with a superheated steam dryer integrated in a local district heat and power plant. Chem Eng J 87(3): 381-394

Bourgault G, Lesage P, Samson R (2012) Systematic disaggregation: a hybrid LCI computation algorithm enhancing interpretation phase in LCA. Int J Life Cycle Assess 17(6):774-786

Collet $P$ et al (2011) Time and life cycle assessment: how to take time into account in the inventory step? In: Finkbeiner M (ed) Towards life cycle sustainability management. Springer; 1 st Edition, Berlin

Collinge WO et al. (2011) Enabling dynamic life cycle assessment of buildings with wireless sensor networks. 2011 I.E. Int Symp Sustain Syst Technol (Issst), 6

Collinge WO et al (2013) Dynamic life cycle assessment: framework and application to an institutional building. Int J Life Cycle Assess 18(3): 538-552

Cucurachi S, Heijungs R, Ohlau K (2012) Towards a general framework for including noise impacts in LCA. Int J Life Cycle Assess 17(4): 471-487

Defourny J, Thorbecke E (1984) Structural path analysis and multiplier decomposition within a social accounting matrix framework. Econ J 94(373):111-136

Dubreuil A, Gaillard G, Müller-Wenk R (2007) Key elements in a framework for land use impact assessment within LCA. Int J Life Cycle Assess 12(1):5-15

Field F, Kirchain R, Clark J (2000) Life-cycle assessment and temporal distributions of emissions: developing a fleet-based analysis. J Ind Ecol 4(2):71-91

Finnveden $\mathrm{G}$ et al (2009) Recent developments in life cycle assessment. J Environ Manag 91(1):1-21

Graedel TE (1998) Streamlined life-cycle assessment. Prentice Hall, Upper Saddle River

Heijungs R, Suh S (2002) The computational structure of life cycle assessment. In: Tukker A (ed) Eco-efficiency in industry and science. 11, 1st edn. Kluwer, Dordrecht, p 241

Hellweg S (2001) Time- and site-dependent life cycle assessment of thermal waste treatment processes. Int $\mathrm{J}$ Life Cycle Assess $6(1): 46$

Kendall A (2012) Time-adjusted global warming potentials for LCA and carbon footprints. Int J Life Cycle Assess 17(8):1042-1049

Kendall A, Price L (2012) Incorporating time-corrected life cycle greenhouse gas emissions in vehicle regulations. Environ Sci Technol 46(5):2557-2563

Kendall A, Chang B, Sharpe B (2009) Accounting for time-dependent effects in biofuel life cycle greenhouse gas emissions calculations. Environ Sci Technol 43(18):7142-7147

Lenzen M (2007) Structural path analysis of ecosystem networks. Ecol Model 200(3-4):334-342

Levasseur A et al (2010) Considering time in LCA: dynamic LCA and its application to global warming impact assessments. Environ Sci Technol 44(8):3169-3174

Mutel CL, Hellweg S (2009) Regionalized life cycle assessment: computational methodology and application to inventory databases. Environ Sci Technol 43(15):5797-5803

Owens JW (1997) Life-cycle assessment in relation to risk assessment: an evolving perspective. Risk Anal 17(3):359-365

Pehnt M (2006) Dynamic life cycle assessment (LCA) of renewable energy technologies. Renew Energy 31(1):55-71

Reap J et al (2008) A survey of unresolved problems in life cycle assessment. Int J Life Cycle Assess 13(4):290-300

Schwartz L (1950) Théorie des distributions, I-II, 1st edn. Hermann \& Cie, Paris, 1950-1951

Schwietzke S, Griffin WM, Matthews HS (2011) Relevance of emissions timing in biofuel greenhouse gases and climate impacts. Environ Sci Technol 45(19):8197-8203 
Shah V, Ries R (2009) A characterization model with spatial and temporal resolution for life cycle impact assessment of photochemical precursors in the United States. Int J Life Cycle Assess 14(4):313-327

Sonnemann G et al (2011) Global guidance principles for life cycle assessment database - "Shonan Guidance Principles". In: Evers D, Kapustka L (eds) SCP documents. UNEP - SETAC, Geneva, p 158

Stasinopoulos P et al (2012) A system dynamics approach in LCA to account for temporal effects - a consequential energy LCI of car body-in-whites. Int J Life Cycle Assess 17(2):199-207
Suh SW, Heijungs R (2007) Power series expansion and structural analysis for life cycle assessment. Int J Life Cycle Assess 12(6): 381-390

Udo de Haes HA et al. (2002) Life-cycle impact assessment: striving towards best practice. In: Society of Environmental Toxicology and Chemistry (SETAC) (ed) Pensacola

Zhai P, Williams ED (2010) Dynamic hybrid life cycle assessment of energy and carbon of multicrystalline silicon photovoltaic systems. Environ Sci Technol 44(20):7950-7955 